\title{
MicroRNA-194 suppresses prostate cancer migration and invasion by downregulating human nuclear distribution protein
}

\author{
QI KONG, XU-SHEN CHEN, TIAN TIAN, XIANG-YOU XIA and PEILIN XU \\ The Key Laboratory of Gene Engineering of the Chinese Ministry of Education, \\ Sun Yat-Sen University, Guangzhou, Guangdong 510275, P.R. China
}

Received April 25, 2016; Accepted June 7, 2016

DOI: $10.3892 /$ or.2016.5305

\begin{abstract}
Human NudC nuclear distribution protein (hNUDC) is differentially expressed between normal and cancer cells. Based on its marked altered expression and its roles in modulating cell division, cytokineses and migration, a detailed understanding of the mechanisms regulating hNUDC expression in cancer cells is critical. In this study, we identified miR-194 as a downstream target of hNUDC and linked its expression to reduced metastatic capacity and tumorigenicity of prostate cancer (PCa) cells. Using miRNA target prediction programs, hNUDC mRNA was found to contain a potential binding site for miR-194 within its 3'UTR. A Reporter assay confirmed that post-transcriptional regulation of hNUDC was dependent on the miR-194 binding site. Forced expression of miR-194 in PCa cell lines, PC-3 and DU-145, led to a decrease in the mRNA and protein levels of hNUDC. Overexpression of miR-194 in these cells inhibited cell migration and invasion, and induced multinucleated cells. Our data showed that hNUDC knockdown by siRNA significantly reduced the migration and invasion in the PC-3 and DU-145 cells, phenocopying the results of miR-194 overexpression. Furthermore, lentivirus-mediated stable expression of miR-194 in PCa cells reduced the ability of colony formation as detected by a soft agar assay and exhibited significantly less tumorigenic ability in vivo. Our results suggest a novel role for miR-194 in effectively controlling cell metastatic processes in PCa cells via the regulation of hNUDC expression.
\end{abstract}

\section{Introduction}

Prostate cancer ( $\mathrm{PCa}$ ) continues to be the most common lethal malignancy diagnosed in men and metastatic progression of $\mathrm{PCa}$ is a major cause of death. During progression to metastasis,

Correspondence to: Professor Peilin $\mathrm{Xu}$, The Key Laboratory of Gene Engineering of the Chinese Ministry of Education, Sun Yat-Sen University, 135 Xingang Xi Road, Guangzhou, Guangdong 510275, P.R. China

E-mail: lssxpl@mail.sysu.edu.cn

Key words: miR-194, hNUDC, prostate cancer, migration, invasion, tumorigenic
PCa cells are thought to acquire a mesenchymal phenotype, which allows them to dissociate from the primary tumor, invade, and migrate to distant organs $(1,2)$. Cell metastasis involves the accumulation of sequential disrupted expression of genes, which in turn promotes cancer progression $(3,4)$. MicroRNAs (miRNAs), a class of small non-coding RNAs, are single-stranded RNAs consisting of 22 nucleotides that negatively regulate expression of their target genes at the 3'-untranslated regions (3'UTRs) (5-7). A growing number of miRNAs have been described as candidate oncogenes or tumor-suppressor genes through respective binding to target tumor-suppressor genes or oncogenic genes (8-11). Over the last two decades, distinct expression profiles of miRNAs have been linked to tumor development and progression in PCa (12-15). Nevertheless, the exact mechanism of multiple molecular events in PCa initiation, growth, invasion and metastasis remains unclear.

The miRNA-194 family is comprised of miR-215, miR-194-1, miR-192 and miR-194-2, which are clustered and expressed as two separate polycistronic pri-miRNA transcripts. The miR-192 and miR-194-2 cluster on chromosome 11q13.1 and the miR-215 and miR-194-1 cluster on chromosome 1q41. miR-194-1 and miR-194-2 have the identical mature sequence. miR-192 and miR-215 are closely related with similar seed sequence. Like many other miRNAs, miR-194 may contribute to the development and progression of different types of cancers, implicating a tumor suppressor function. Emerging evidence suggests that miR-194 is downregulated in a number of different malignancies, such as colorectal cancer (16), renal childhood neoplasms (17), liver (18) and endometrial cancer (19). Conversely, increased miR-194 expression is found in a range of cancer cellss, including those of gastric (20), colorectal (21) and endometrial cancer (22) and is associated with reduced cancer metastasis. However, the biological functions of the deregulation of miR-194 in tumor progression have not yet been completely defined.

The mammalian NudC nuclear distribution protein (NUDC) is expressed in a broad range of tissues and is increasingly being recognized as a multifunctional protein that affects various cellular responses, such as cell division (23), proliferation (24), migration $(25,26)$, and cytokinesis $(27,28)$. Aberrant regulation of hNUDC expression is correlated with a variety of pathologies. For example, the expression levels of hNUDC are much higher in erythroid precursor cells 
compared to other human tissues (29). In addition, hNUDC protein expression was found to be significantly upregulated in patients with acute myelogenous and acute lymphoblastic leukemia compared to aspirates from normal controls $(30,31)$. Furthermore, hNUDC was found expressed in neuroectodermal tumors, but not in non-neoplastic brain tissue (32). There is also an inverse correlation between hNUDC expression and nodal metastasis in esophageal cancer (33). Downregulation of human hNUDC mRNA, including the use of the antisense oligonucleotides and small interfering (si)RNAs resulted in impairment of both cell proliferation in various types of cancer in vitro $(27,34)$. As a consequence, the cells exhibited nuclear enlargement and multiple nuclei, possibly due to a failure to complete the inhibition of both mitosis and cytokinesis. As may be expected, a protein involved in mitotic cell division is also found to play a role in cancer. According to previous studies in the literature, adenovirus-mediated overexpression of hNUDC in LNCaP, DU-145 and PC-3 cells significantly attenuatedthe rate of cell proliferation through $\mathrm{G} 2 / \mathrm{M}$ phase arrest. This has been interpreted as a potential requirement for hNUDC to accomplish the first steps of metastasis (35). Previous results have demonstrated that both upregulation and downregulation of hNUDC play an important role in anticancer intervention.

In the present study, the regulation of biological functions by miR-194 was identified for the first time in PCa by the targeting of the hNUDC gene. Transfection of DU-145 and PC-3 cell lines with miR-194 decreased hNUDC mRNA and protein expression and reduced cell migration and invasion. In vitro and in vivo experiments indicated that the overexpression of miR-194 suppressed colony formation and tumorigenicity of PCa cells in nude mice. These findings suggest that miR-194 may act as a tumor suppressor in $\mathrm{PCa}$, which is consistent with its role in other human cancers.

\section{Materials and methods}

Cell lines and culture conditions. Human prostate cancer cell lines, PC-3 and DU-145, were obtained from the American Type Culture Collection (ATCC; Manassas, VA, USA). Cells were grown in Dulbecco's modified Eagle's medium (DMEM; Gibco ${ }^{\circledR}$ by Life Technologies ${ }^{\mathrm{TM}}$, Grand Island, NY, USA) supplemented with $10 \% \mathrm{v} / \mathrm{v}$ inactivated fetal bovine serum (FBS; Biological Industries Israel Beit Haemek, Ltd., Kibbutz Beit-Haemek, Israel) and $100 \mathrm{U} / \mathrm{ml}$ penicillin + $100 \mu \mathrm{g} / \mathrm{ml}$ streptomycin. Cells were maintained in a humidified incubator at $37^{\circ} \mathrm{C}$ with $5 \% \mathrm{CO}_{2}$.

Transfection procedures. Cells were plated at $1 \times 10^{5}$ in a 6 -well plate. Twelve hours later, cells were transfected with $50 \mathrm{nM}$ of scrambled control, miR-194, miR-194 inhibitor, inhibitor control or siRNA for hNUDC (Suzhou GenePharma Co., Ltd., Suzhou, China) with the use of Lipofectamine ${ }^{\circledR} 2000$ reagent (Invitrogen by Life Technologies, Carlsbad, CA, USA). siRNA for hNUDC used here has been previously described (27). After transfection, the cells were processed for western blot analysis, quantitative real-time PCR (RT-qPCR), migration and invasion assays.

RNA isolation and RT-qPCR. Total RNA was isolated from the prostate cell lines using TRIzol reagent, and quantified spectrophotometrically. The reverse transcription of miRNAs from total RNA $(1 \mu \mathrm{g})$ was performed with miR-194-specific stem-loop primer (5'-CTCAACTGGTGTCGTGGAGTCG GCAATTCAGTTGAGTCCACATG-3') using a Bestar ${ }^{\text {TM }}$ qPCR RT kit (DBI ${ }^{\circledR}$ Bioscience, Ludwigshafen, Germany). To measure hNUDC mRNA expression levels, the first strand of cDNA was synthesized using oligo (dT) primers by Bestar ${ }^{\mathrm{TM}}$ qPCR RT kit and the reverse transcription product was amplified using Bestar ${ }^{\mathrm{TM}}$ SybrGreen qPCR Mastermix (DBI ${ }^{\circledR}$ Bioscience). The sequences of the primers specific for hNUDC were 5'-CAGTGGGGTCTTGCTGTCATCT-3' (forward) and 5'-CTAACCCTTGCCTTTCAACTCA-3' (reverse). Quantitative PCR was performed using the StepOnePlus ${ }^{\mathrm{TM}}$ Real-Time PCR System (Applied Biosystems). The PCR reaction consisted in a denaturation step at $95^{\circ} \mathrm{C}$ for $10 \mathrm{~min}$, followed by 40 amplification cycles at $95^{\circ} \mathrm{C}$ for $10 \mathrm{sec}$, and annealing at $60^{\circ} \mathrm{C}$ for $35 \mathrm{sec}$ and extension at $72^{\circ} \mathrm{C}$ for $30 \mathrm{sec}$. Gene expression was normalized using endogenous $\beta$-actin or U6 as a control. Densitometric quantification was perfomed with the comparative cycle threshold method $\left(2^{-\Delta \Delta \mathrm{C} t}\right)(36)$.

Western blot analysis. Total lysates from DU-145 and PC-3 cells $(10 \mu \mathrm{g})$ were applied to $10 \%$ SDS-PAGE and transferred onto polyvinylidene difluoride (PVDF) membranes. Membranes were blocked with 5\% BSA in 1X Tris-buffered saline containing $0.1 \%$ Tween-20 (TBST) for $1 \mathrm{~h}$ at room temperature. Western blot analysis was performed by incubating the membranes with primary antibodies for hNUDC (Abcam, Cambridge, MA, USA) at a dilution of 1:3,000 in 5\% BSA in 1X TBST. After overnight incubation at $4^{\circ} \mathrm{C}$, the membranes were incubated with goat anti-mouse IgG alkaline phosphatase (AP) conjugated secondary antibodies (Cell Signaling Technology, Inc.) at 1:1,000 for $1 \mathrm{~h}$ at room temperature. Signals were visualized using an enhanced chemiluminescence (ECL) detection system (Pierce, Rockford, IL, USA). Densitometric quantification was performed using ImageJ software.

Dual-Luciferase assay. 3'UTR mRNA sequence of hNUDC containing a miR-194 binding site was amplified by the following primers: 5'-ATTACGCGTCCCCTGTTTTTTC CTCCCTG-3' (forward) and 5'-CGGAAGCTTAGCTGGGC AAATCGTTTTAA-3' (reverse). The PCR product was then cloned downstream to the luciferase gene in plasmid pMIR-Report. A mutation construct was made by deletion of several bases within the binding site and cloned into the same vector. The control vector was used for normalization of cell number and transfection efficiency. Co-transfection of synthetic miR-194 and luciferase report constructs were transfected in HEK-293T cells by using Lipofectamine 2000 reagent. Luciferase assays were performed using the DualLuciferase ${ }^{\circledR}$ Reporter Assay System kit (Promega Corp., Madison, WI, USA) and luminescence was measured on a GloMax $^{\circledR} 96$ microplate luminometer (Promega).

In vitro invasion and migration assays. Cellular invasion and migration were assessed using the Cultre ${ }^{\circledR}$ Basement Membrane Extract(BME),PathClear ${ }^{\circledR}$ (Trevigen, Gaithersburg, MD, USA) according to the manufacturer's protocol. Cells at a density of $5 \times 10^{4}$ were seeded onto Transwell ${ }^{\circledR}$ Permeable 
Supports (Corning Incorporated, Corning, NY, USA). For the migration assay, the plates were incubated for $12 \mathrm{~h}$ at $37^{\circ} \mathrm{C}$. The cells that migrated through the pores without the matrix to the lower surface of the membrane were fixed in $70 \%$ ethanol and stained with crystal violet. For the invasion assay, the plates were incubated for $48 \mathrm{~h}$ and the cells that invaded through the pores covered with the matrix to the lower surface of the membrane were fixed and stained. The cells were counted under a light microscope in five separate fields. Each blue point indicated an individual cell and all the images were scanned and counted at $\mathrm{x} 40$ magnification.

Determination of nuclear morphology. Cells were transfected for $48 \mathrm{~h}$ and fixed with methanol prior to being stained with May-Grünwald-Giemsa staining solution for $15 \mathrm{~min}$ at room temperature. The stained slides were examined by a phase contrast microscope at $\mathrm{x} 40$ magnification.

Generation of stable cell lines. The packaged lentivirus expressing miR-194, anti-miR-194-sponge and GFP-control were purchased directly from ViGene Biosciences (Shandong, China). Stable expression of miR-194, GFP-control and anti-miR-194-sponge in PC-3 and DU-145 cells was achieved through selection with $2 \mu \mathrm{g} / \mathrm{ml}$ puromycin (Amresco, Solon, $\mathrm{OH}, \mathrm{USA})$.

Clonogenic cell growth assay. The clonogenic cell growth of PC-3 and DU-145 cell lines was examined on soft agar using Cell Transformation Detection Assay (Millipore, Temecula, CA, USA). Briefly, each well of a 24-well plate was first layered with $0.8 \%$ agarose in growth medium (DMEM supplemented with $10 \%$ FBS). The cell lines to be tested were trypsinized, and $1 \times 10^{4}$ cells were resuspended in a growth medium containing $0.4 \%$ agarose and then they were poured as a top layer in the 24-well plates. The plates were incubated at $37^{\circ} \mathrm{C}$ for 28 days. Colony formation was observed using Cell Stain Solution overnight and the diameter of colonies was scored under a microscope at $\mathrm{x} 40$ magnification. Cells were counted with the Cell Quantification Solution after a 4-h incubation at $37^{\circ} \mathrm{C}$, followed by a spectrophotometer reading at $\mathrm{OD}_{490}$.

Nude mouse xenograft assay. The female BALB/c nude mice (18 g, aged 6 weeks) were obtained from Guangdong Medical Laboratory Animal Center (permit no. SCXK 2013-0002). Animals were maintained and cared for in the Traditional Chinese Medicine and Marine Drugs Laboratory of Sun Yat-Sen University (permit no. SYXK 2014-0020) which conformed to the Guide for the Care and Use of Laboratory Animals. In vivo experiments were performed in accordance with a protocol approved by the Animal Ethics and Welfare Committee of Sun Yat-Sen University. PC-3 and DU-145 cell lines stably expressing miR-194, anti-miR-194-sponge or GFP-control were suspended in a PBS buffer at a concentration of $1 \times 10^{7}$ cells $/ \mathrm{ml}$. Cell suspension $\left(2 \times 10^{6}\right.$ cells/mouse $)$ were injected subcutaneously below the right scapula of the BALB/c-nude mice. Seven mice from each of the three groups were used. The experiment ended when the tumor volume reached $2,000 \mathrm{~mm}^{3}$. The tumors were measured with microcalipers three times a week and the volumes were calculated

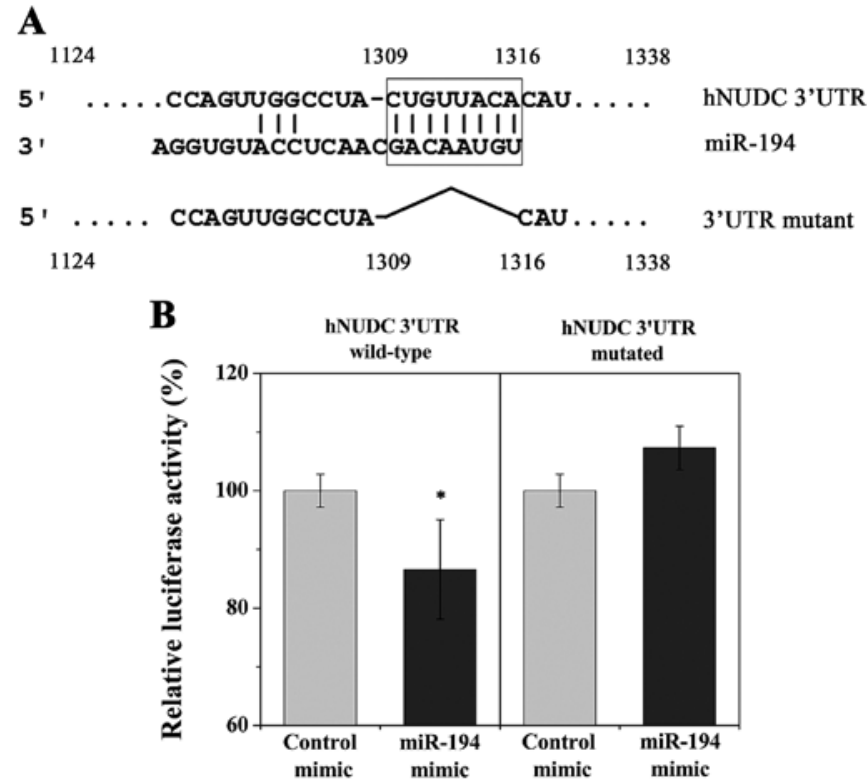

Figure 1. The 3'UTR of human NudC nuclear distribution protein (hNUDC) contains a single predicted miR-194 target site. (A) Schematic representation of firefly luciferase reporter constructs containing the 200-nt 3'UTR of hNUDC. In a mutated construct, a miR-194 target site was disrupted by deletion of eight nucleotides within the seed sequence as indicated. (B) Luciferase reporter assays using HEK-293T cells co-transfected with the reporter gene constructs described above with miR-194 or control mimic. Forty-eight hours after transfection, luciferase activity was measured. Activity was normalized to Renilla luciferase activity. Data for each reporter construct are expressed relative to control transfected cells. Data are expressed as the means $\pm \mathrm{SE}$. $(\mathrm{n}=3) .{ }^{*} \mathrm{P}<0.05$.

as $\mathrm{V}\left(\mathrm{mm}^{3}\right)=$ length $(\mathrm{mm}) \times(\text { width }(\mathrm{mm}))^{2} \times 0.5$. The mice were euthanized on day 30 and the tumors were harvested and weighed.

Significance testing. Statistical relevant differences were assigned to a p-value of at least $\leq 0.05$ using an unpaired Student's t-test in all functional assays and densitometric analyses of western blot analysis assays. All data represent at least three independent experiments. Results are expressed as the mean \pm standard error $(\mathrm{SE})$.

\section{Results}

hNUDC is a direct target of miR-194. To determine whether hNUDC can be regulated by miRNAs, we used TargetScan $(37,38)$ (http://www.targetscan.org/) and miRanda (39-41) (http://www.microrna.org/) to predict miRNAs that could potentially target the hNUDC 3'UTR. A potential binding site for miR-194 was identified at the hNUDC 3'UTR from nucleotides 1309-1316 (Fig. 1A). To validate hNUDC as a direct target of miR-194, both wild-type and mutant hNUDC 3'UTRs were cloned downstream of the firefly luciferase ORF in a pMIR-Report vector (Fig. 1A). The wild-type and mutant hNUDC 3'UTRs luciferase expression vectors were co-transfected with scrambled control or miR-194 mimic into HEK-293T cells. Relative luciferase activity was significantly reduced for wild-type hNUDC 3 'UTR, indicating that hNUDC is a potential direct target of miR-194 (Fig. 1B). Mutation in the predicted miR-194 target site abrogated 

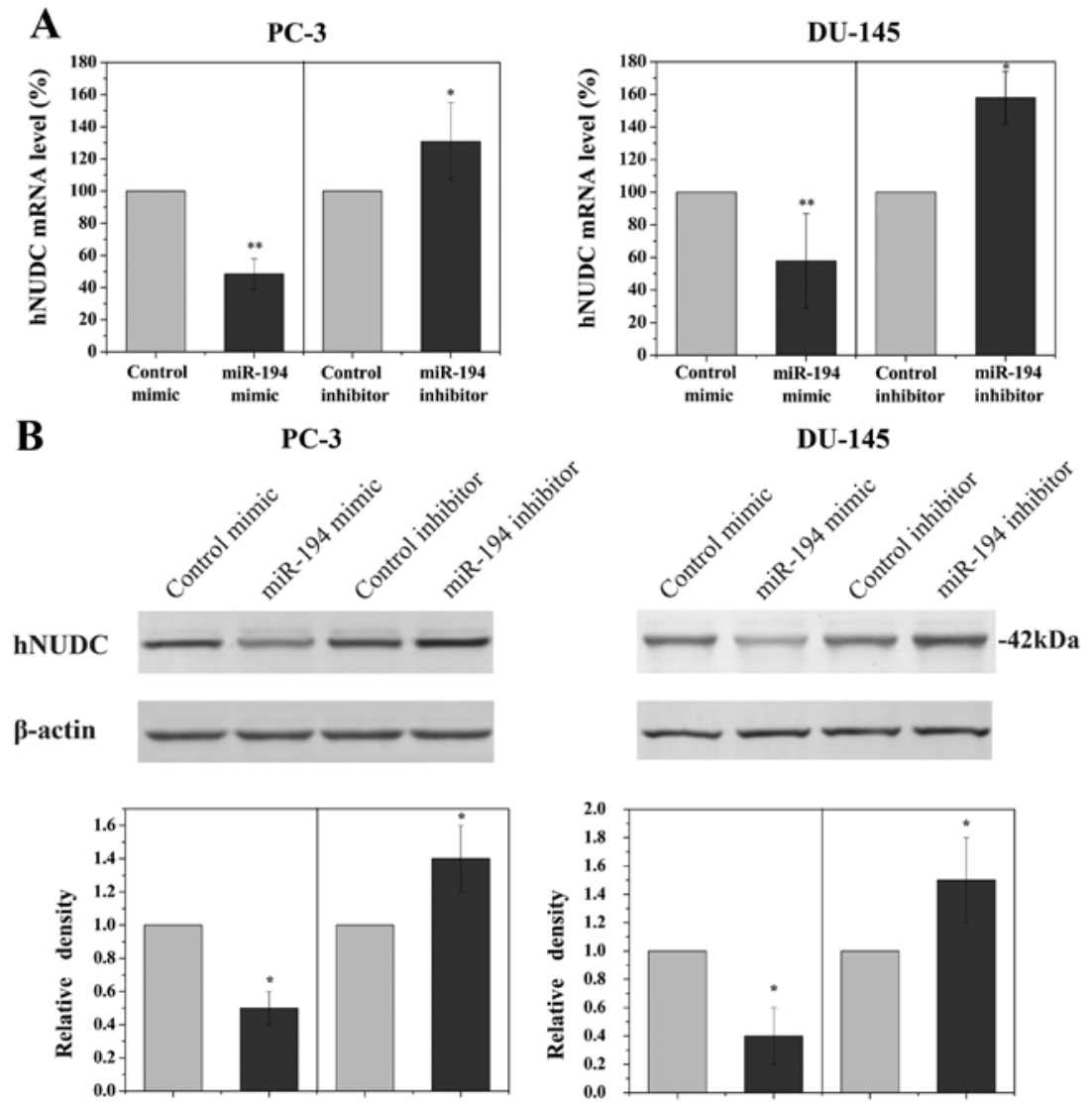

Figure 2. Validation of human NudC nuclear distribution protein (hNUDC) as a direct target of miR-194. (A) Inverse correlation between the expression of miR-194 and hNUDC at miRNA levels in the indicated cell lines. Cells were transfected with scrambled control, miR-194, miR-194 inhibitor or inhibitor control for $48 \mathrm{~h}$ and RT-qPCR was used to confirm the hNUDC transcript levels. (B) Inverse correlation between the expression of miR-194 and hNUDC at protein levels in the indicated cell lines. hNUDC was detected by western blot analysis; $\beta$-actin was used as a loading control. Data are expressed as the means \pm SE. $(\mathrm{n}=3) .{ }^{*} \mathrm{P}<0.05 ;{ }^{* *} \mathrm{p}<0.001$.

inhibition by miR-194 mimic, confirming the functionality of this target site (Fig. 1B).

hNUDC expression levels are regulated by miR-194 in prostate cancer cells. To determine if the hNUDC gene was a biologically relevant target of miR-194 in PCa cells, PC-3 and DU-145 cell lines were transfected with miR-194 mimic or miR-194 inhibitor. To demonstrate the specificity of miR-194 in order to evaluate the magnitude of the changes in hNUDC mRNA levels, non-targeting miRNA inhibitor and non-targeting scrambled miRNA were used as controls. qRT-PCR analyses revealed a significant reduction in the RNA expression of hNUDC following transfection with miR-194 mimic, whereas miR-194 inhibitor increased hNUDC levels (Fig. 2A). Western blot analysis was also employed to examine the hNUDC protein, showing a prominent decrease in the expression of hNUDC when PC-3 and DU-145 cells were transfected with miR-194 mimic. Conversely, inhibiting the endogenous miR-194 in PC-3 and DU-145 cells using miR-194 inhibitor increased the hNUDC protein level (Fig. 2B).

miR-194 attenuates the migration and invasion of prostate cancer cells. To date, migration and invasion are known as the key processes in many cancers. In order to investigate whether the ectopic overexpression of miR-194 is involved in these processes, we assessed the in vitro migratory and invasive capacities by Transwell migration assay. The ectopic expression of miR-194 decreased the invasiveness of PC-3 and DU-145 cells compared to the control mimic-transfected cells (Fig. 3A). Upon inhibition of endogenous miR-194 by miR-194 inhibitor, a visible opposite result was seen than in the control inhibitor transfected cells (Fig. 3A). Microscopy results revealed that upon transfection of miR-194 mimic, the loss of migratory capability was accompanied by a loss of cell elongation (Fig. 3B). These findings indicate that upregulation of miR-194 inhibits cell invasion and migration.

Overexpression of miR-194 induces multinucleated cells. Since hNUDC is considered an important mediator of cell proliferation and cytokinesis, thus, it is expected that the inhibition of hNUDC would result in the failure of cell growth and give rise to cells with multiple nuclei. However, our cell proliferation and cell cycle analyses revealed that transfection of the miR-194 mimics into two PC-3 and DU-145 cell lines showed no influence on cell proliferation and cell cycle compared to the scrambled control (data not shown). Morphologically, transfection of cells with the miR-194 resulted in an increase in multinucleated cells coupled with abnormally large, flattened and accumulated multiple nuclei, whereas cells transfected with the mimic and inhibitor controls had normal nuclear morphology (Fig. 4A and B). 

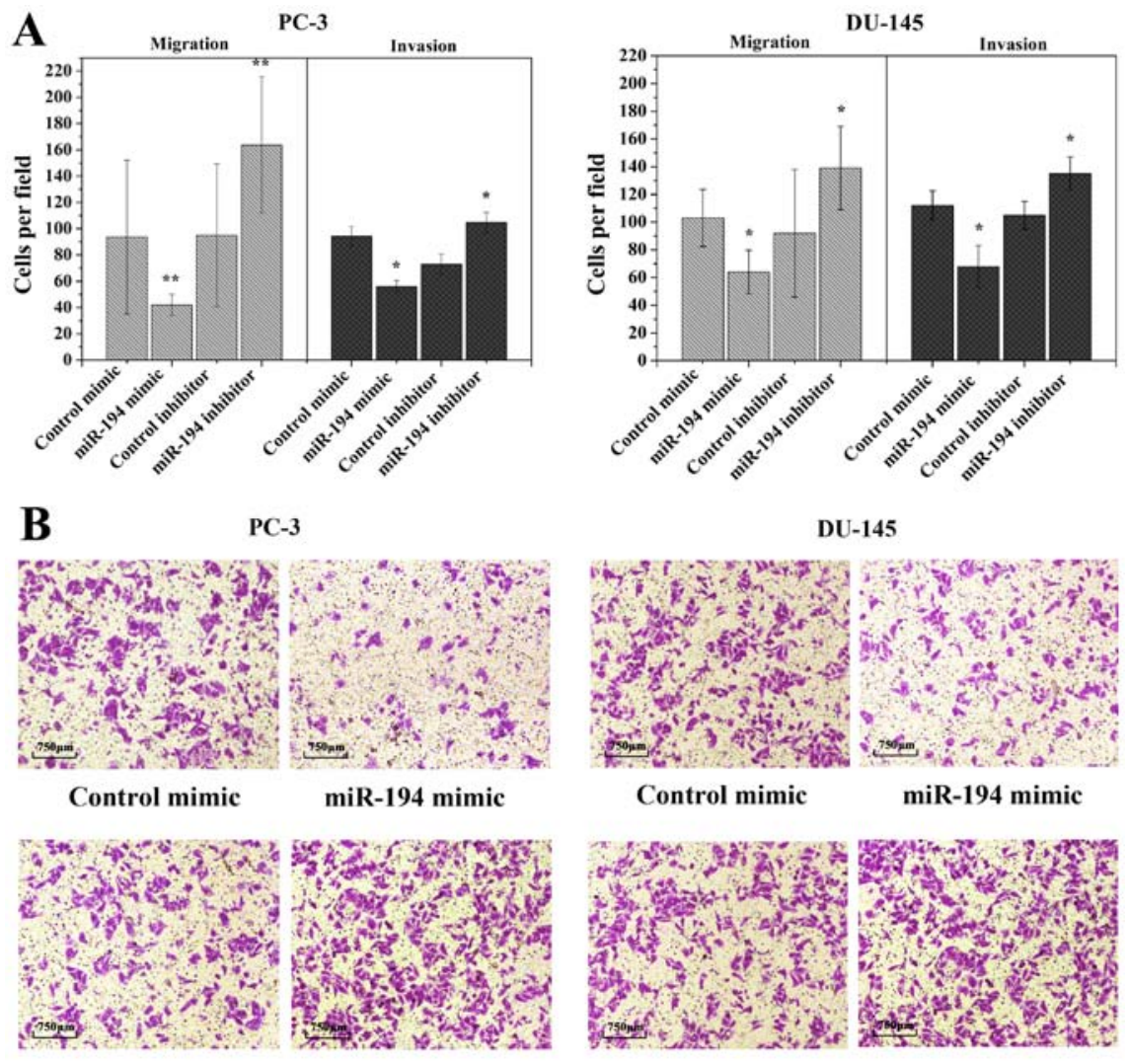

Control mimic miR-194 mimic

Control inhibitor miR-194 inhibitor

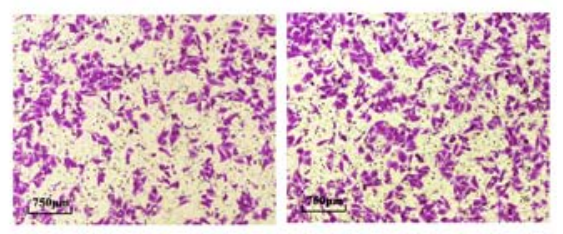

Control inhibitor miR-194 inhibitor

Figure 3. miR-194 affects the cell migratory and invasive potential in vitro. (A) Migration and invasion of PC-3 and DU-145 cell lines transfected with scrambled control, miR-194, miR-194 inhibitor or inhibitor control as depicted by a Transwell migration assay after 12 and $48 \mathrm{~h}$, respectively. Data are expressed as the means \pm SE. $(n=3)$. ${ }^{*} \mathrm{P}<0.05$; ${ }^{* * *} \mathrm{p}<0.001$. (B) Morphological alterations in PC-3 and DU-145 cells upon the indicated transfection assessed by phase-contrast microsopy. Scale bars, $750 \mu \mathrm{m}$.
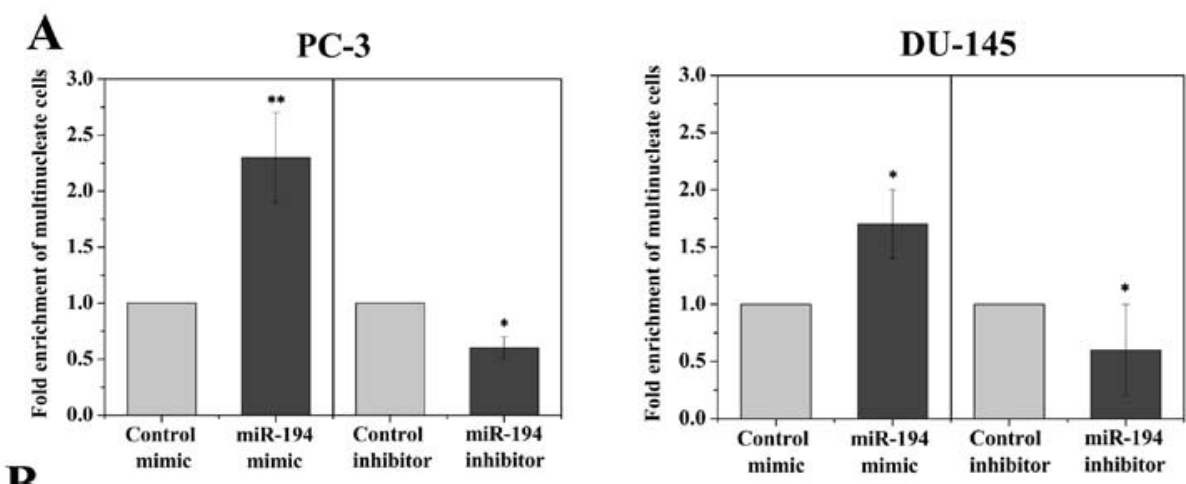

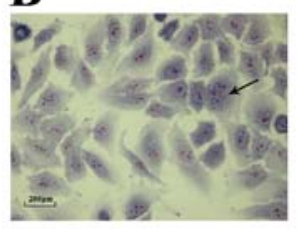

Control mimic

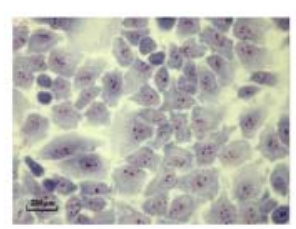

Control inhibitor

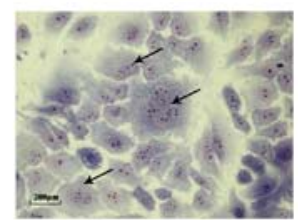

miR-194 mimic

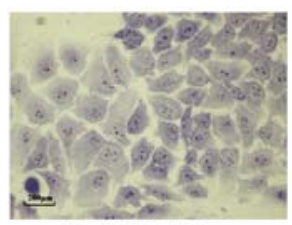

miR-194 inhibitor

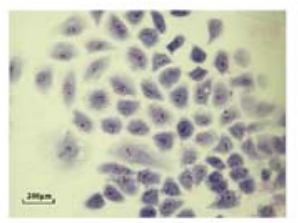

Control mimic

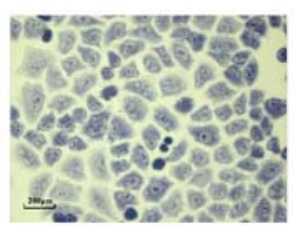

Control inhibitor

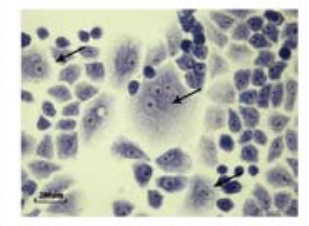

miR-194 mimic

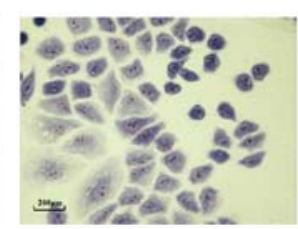

miR-194 inhibitor

Figure 4. Overexpression of miR-194 induces cell cytokinesis. PC-3 and DU-145 cells were transfected with scrambled control, miR-194, miR-194 inhibitor or inhibitor control for $48 \mathrm{~h}$. (A) The number of multinucleated cells was counted at $48 \mathrm{~h}$ after May-Grünwald-Giemsa staining. The columns represent the mean \pm SE of three replicates. ${ }^{*} \mathrm{P}<0.05 ;{ }^{* *} \mathrm{p}<0.001$. (B) Representative morphology of multinucleated cells as assessed by phase-contrast microsopy. Scale bars, $200 \mu \mathrm{m}$. 

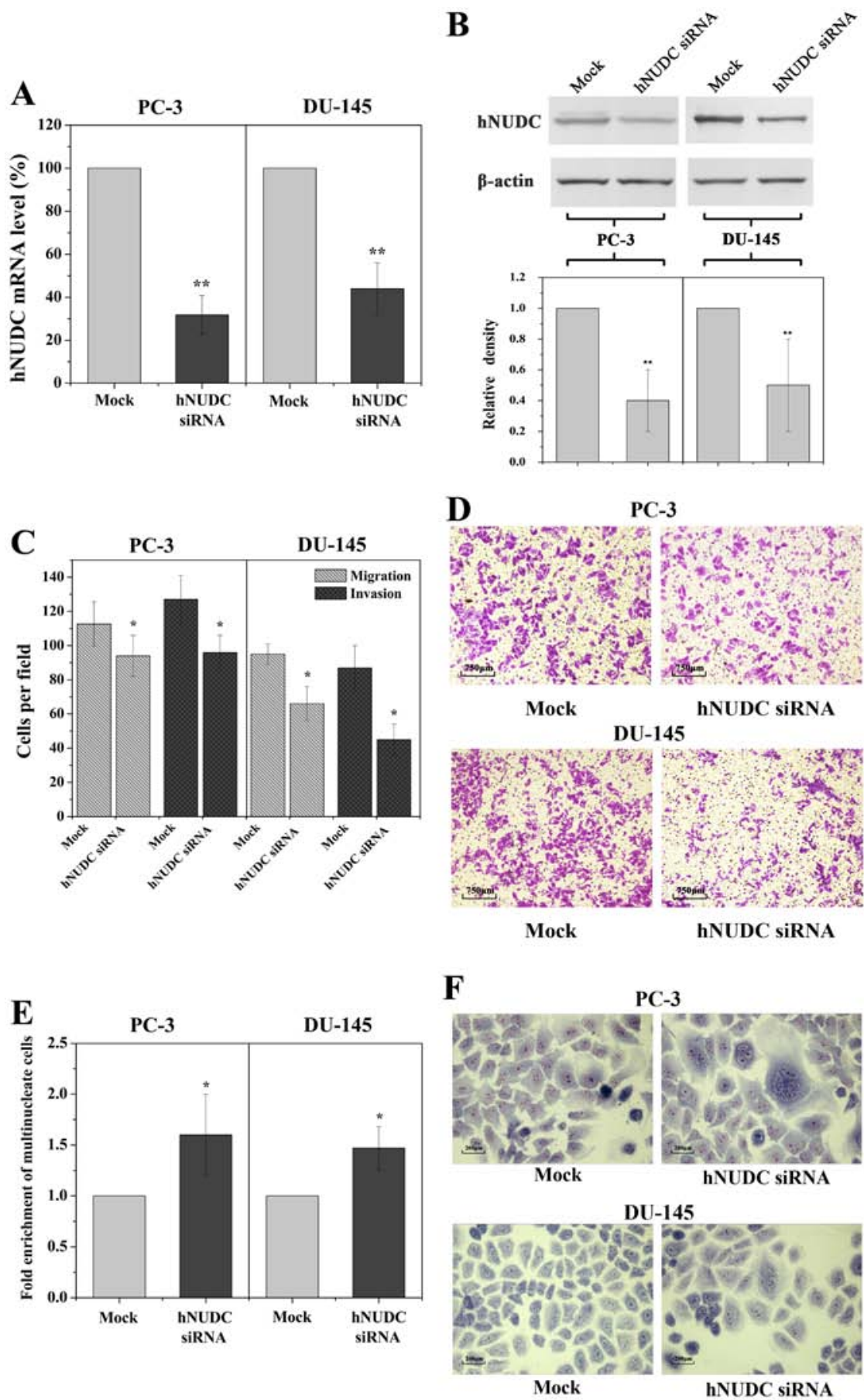

Figure 5. RNA interference-mediated functional effects of hNUDC. (A) PC-3 and DU-145 cells were transfected with hNUDC siRNA and RT-qPCR analysis demonstrated significant reductions in the expression levels of hNUDC mRNA compared to the mock control $48 \mathrm{~h}$ post-transfection. (B) Western blot analysis also revealed significant reduction in the protein levels of hNUDC $48 \mathrm{~h}$ post-transfection with hNUDC siRNA. (C) hNUDC siRNA decreased cell invasion and migration when compared with the mock control. Data are expressed as the means $\pm \mathrm{SE}$. $(\mathrm{n}=3) .{ }^{*} \mathrm{P}<0.05 ;{ }^{* *} \mathrm{p}<0.001$. (D) The effect of hNUDC knockdown on the cellular morphology. Scale bars, $750 \mu \mathrm{m}$. (E) The number of the multinucleated cells formed by the indicated transfection of PC-3 and DU-145 cells after $48 \mathrm{~h}$. The columns represent the mean $\pm \mathrm{SE}$ of three replicates. ${ }^{*} \mathrm{P}<0.05 ;{ }^{* *} \mathrm{p}<0.001$. (F) Representative morphology of multinucleated cells as assessed by phase-contrast microsopy. Scale bars, $200 \mu \mathrm{m}$.

siRNA-mediated inhibition of hNUDC mimics the phenotypes induced by $m i R-194$. The aforementioned results demonstrated that miR-194 is a potent suppressor of cell migration and invasion through the targeting of hNUDC mRNA. Thus, we reasoned that siRNA-mediated knockdown of hNUDC should result in a cell phenotype similar to that of miR-194 overexpression. Indeed, by using hNUDC siRNA we were able to obtain a decrease in the expression of the hNUDC gene, reducing
mRNA levels by 3.3 -fold in the PC- 3 cells and by 2.5 -fold in the DU-145 cells, as determined by qRT-PCR analysis $48 \mathrm{~h}$ post-transfection (Fig. 5A). This result was confirmed by a significant reduction in hNUDC at the protein level (Fig. 5B). Similarly, miR-194 overexpression, significantly reduced the migration and invasion potential following knockdown of hNUDC expression (Fig. 5C and D). Following transfection with hNUDC siRNA, we observed that the multinucleated 

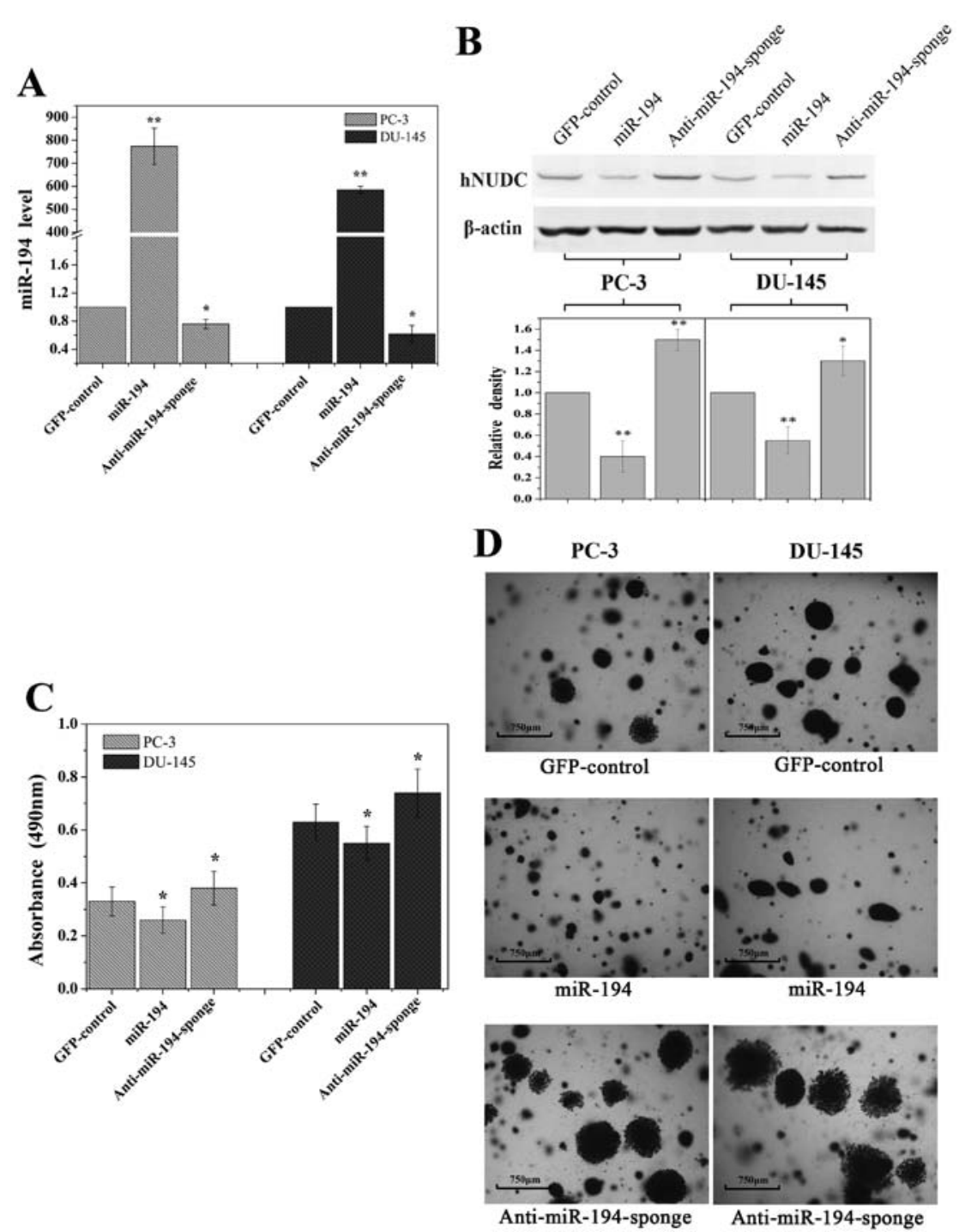

Figure 6. Overexpression of miR-194 reduces colony formation. PC-3 and DU-145 cell lines stably transfected with miR-194, anti-miR-194-sponge or the GFP-control plasmid were cultured for 30 days. (A) The expression levels of miR-194 were confirmed by RT-qPCR. (B) The protein levels of hNUDC were confirmed by western blot analysis. (C) The number of cells were detected at 30-days post-transfection. The bars represent the mean \pm SE of the number of colonies from three independent experiments. ${ }^{*} \mathrm{P}<0.05$; ${ }^{* *} \mathrm{p}<0.001$. (D) The colonies were visualized after staining with crystal violet. Scale bars, $200 \mu \mathrm{m}$.

cells were enhanced following inhibition of endogenous hNUDC (Fig. 5E and F). These results strongly suggest that downregulation of hNUDC expression promotes invasion and migration of prostate cancer cells.

Overexpression of miR-194 inhibits cell growth potential. To examine whether miR-194 regulates primary tumor growth, a standard colony formation assay was used for these studies. We overexpressed miR-194 in PC-3 and DU-145 cells by transduction with high-titer lentivirus expressing miR-194 or anti-miR194-sponge using an empty vector as a control. An 700-fold increase of miR-194 expression was detected by qRT-PCR in the transduced cells, in comparison to cells that were transduced with a control vector expressing GFP (Fig. 6A). As expected, a small decrease in miR-194 in the lentiviral expressing anti-miR-194-sponge was observed (Fig. 6A). The expression level of hNUDC protein was significantly decreased in response to ectopic miR-194 expression (Fig. 6B). Overexpression of miR-194 decreased the total number of cells observed when compared with the control (Fig. 6C). In contrast, anti-miR-194-sponge did not suppress cell growth in soft agar (Fig. 6C). Moreover PC-3 and DU-145 cells transfected with miR-194 formed smaller colonies compared to larger disseminated colonies formed by the GFP-control and anti-miR-194-sponge transduced cells after 30 days (Fig. 6D). The significant reduction of colonies suggests that miR-194 exhibits a tumor-suppressive function in cancer cells.

Overexpression of miR-194 suppresses the growth of $\mathrm{PCa}$ xenografts. We next examined the effect of ectopic miR-194 expression on prostate cancer tumorigenesis in vivo. DU-145 cells stably expressing either miR-194 or anti-miR-194-sponge was subcutaneously injected into 6-week-old nude mice. The control group was injected with DU-145 stably expressing GFP. Tumor growth was monitored twice weekly for 30 days. As shown in Fig. 7, tumors derived from cells expressing antimiR-194-sponge grew more rapidly. By the end of the study, the tumors were larger (tumor volume) and heavier (tumor weight) than the GFP-control. In contrast, tumors derived from 

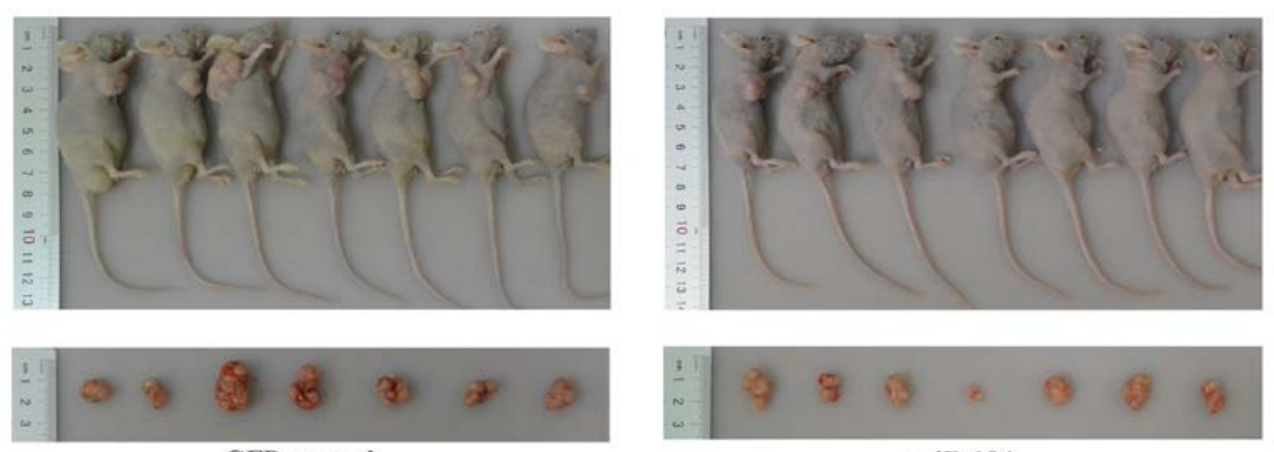

GFP-control

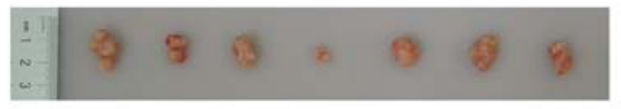

miR-194
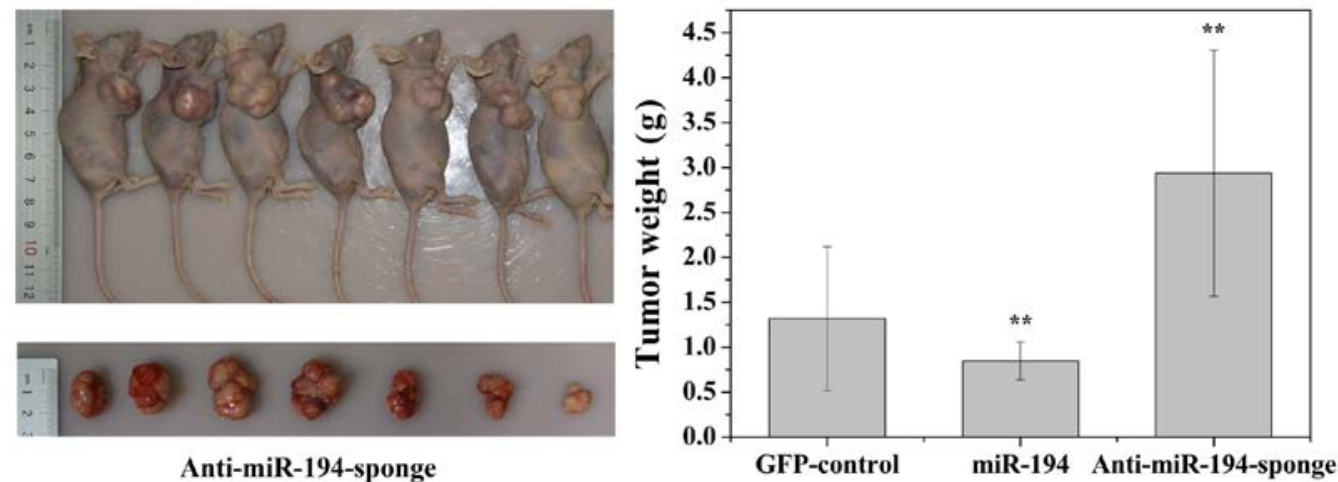

Anti-miR-194-sponge

GFP-control miR-194 Anti-miR-194-sponge

Figure 7. Overexpression of miR-194 suppresses xenograft tumor growth. DU-145 cells stably transfected with miR-194, anti-miR-194-sponge or the GFP-control plasmid were injected subcutaneously into nude mice. The tumors were grown to the indicated size and the mice were randomly divided into three groups (7 mice/group). The tumor size and body weight were measured on day-30. Data are represented as the mean $\pm \mathrm{SE}$ of each group. ${ }^{*} \mathrm{P}<0.001$.

the cells with miR-194 overexpression appeared significantly smaller than the tumors formed from the cells transduced with the GFP-control.

\section{Discussion}

Little is known regarding the role of hNUDC in cancer. Previous results have demonstrated that hNUDC is ubiquitously expressed in normal human tissues. However, its protein levels are markedly overexpressed in almost all types of cancer cells, including cutaneous T-cell lymphoma and neuroectodermal tumors (29-33). Human NUDC has been identified in various molecular contexts accounting for its numerous aliases: a dynein-associated nuclear movement protein (27), chaperone protein (42), neuronal migration protein (43). Hence, hNUDC is a multifunctional protein and is involved in diverse biological events. Our laboratory has recently reported that hNUDC acts as a secondary ligand for thrombopoietin receptor $(\mathrm{Mpl})$ involved in regulating proliferation and differentiation of different types of megakaryocyte cells (44-46). However, a complete understanding of all of the hNUDC functions remains unclear since this protein interacts with a number of other proteins with multi-functional effects. The present study is the second study concerning hNUDC in prostate cell lines. Our study focused on ectopic expression of miR-194 and its effects on prostate cancer by suppressing hNUDC expression. The preliminary data indicated that downregulation of hNUDC by miR-194 or si-hNUDC in DU-145 and PC-3 cells did not induce cell proliferation or cell cycle progression (data not shown) but rather resulted in morphological changes usually associated with an enhanced multinucleated phenotype. However, suppression of colony formation in the soft agar assay in the PC-3 and DU-145 cells suggests that hNUDC may play a role in the inhibition of tumor cell growth. We also observed that downregulation of endogenous hNUDC impaired prostate tumor cell migration and invasion with either miR-194 or siRNA strategies, although the mechanism by which this effect is mediated remains to be elucidated. To demonstrate that miR-194 contributes to $\mathrm{PCa}$ development in vivo, we employed a xenograft nude mouse model and found that primary tumor growth was reduced subcutaneously. Thus, it is possible that miR-194 may play a more general role in suppressing oncogenic processes in prostate cancer. This conclusion is further supported by the overexpression of miR-194 whereby it reduced protein bone morphogenetic protein 1 (BMP1) levels in PC-3 cells causing a significant decrease of invasion in Matrigel-coated Transwell chambers (47). During prostate cancer progression, most deaths from prostate cancer are not due to the primary tumor but rather to secondary metastases to distant organs. For this reason it is of fundamental importance to study the mechanisms that drive prostate cancer invasion and metastases. These data support the biological relevance of model systems implicating miR-194 and hNUDC expression as potential clinical markers.

Although it goes beyond the scope of this study, we initially tested five cell lines, RWPE-1 and WPMY-1 which are classically defined as non-tumoral, and the cancer cell lines LNCaP, DU-145 and PC-3. We found that RWPE-1, WPMY-1 and $\mathrm{LNCaP}$ inherently display a relative higher expression of 
miR-194 compared to the DU-145 and PC-3 cells. However, the inverse relationship between the hNUDC and miR-194 expression levels was observed in all the normal and prostate tumor cell lines (data not shown). Both the DU-145 and PC-3 cell lines, derived from secondary metastatic prostate tumors, were used in our study as it remains possible that low expression of miR-194 is associated with acquisition of a more metastatic phenotype. We did not find any correlation between hNUDC expression and miR-194 in clinical parameters used to assess the poor prognosis of prostate cancer. In a future study, we will address this issue in more depth, since this issue remains to be investigated in a larger series of cases.

Recently, miR-194 expression profiles have been detected in a variety of tumor entities and it has been suggested that miRNAs are mostly downregulated in these cancer cells, although some are overexpressed, playing a critical role in tumor initiation and progression (16-19). One of the largest qRT-PCR analyses of tissue cohorts reported by Selth et al, indicated that miR-194 was robustly expressed in malignant prostate tissue and its expression in primary tumors was associated with a poor prognosis (48). One question that arises from this result is the mechanism that is involved in the increased upregulation in malignant prostate tissues compared to the matched poor prognosis tissues. As prostate tumors are very heterogeneous, the relative higher levels of expression of the miRNAs may be masked by the contribution of the stromal levels of miR-194 that may remain elevated. In addition, the miR-194 family consists of four members whose clusters are located on two separate chromosomes, and we believe that an explanation for this discrepancy is likely due to the fact that their spatial and temporal expression is tightly regulated by two genomic loci.

Although hNUDC is identified as a target of miR-194, it is a well accepted fact that an miRNA can target many genes. Database mining revealed that a single miR-194 may have multiple targets in tumorigenesis. One study implicated ectopic overexpression of miR-194 in the regulation of colon cancer angiogenesis in vivo, by suppressing its target, thrombospondin-1 (49). Moreover, upregulation of miR-194 induced downregulation of RBX1 showing significant inhibition of tumor size, invasion and tumor node metastasis (50). Similarly, BMI-1 knockdown inhibited cell proliferation and clone growth and BMI-1 was recently proposed as a biologically relevant miR-194 target in endometrial cancer cells (22). Furthermore, miR-194 was found to inhibit chondrogenic differentiation of human adipose-derived stem cells by targeting Sox 5 and suppressed osteosarcoma cell proliferation and metastasis in vitro (51) and in vivo by targeting $\mathrm{CDH} 2$ and IGF1R (52). Therefore, we cannot exclude the possibility that these candidate targets for miR-194 other than hNUDC may be involved in tumor suppression. The activity of hNUDC as a cancer candidate target for miR-194 needs to be confirmed in future studies.

In conclusion, our study is the first to indicate that miR-194 interacts directly with hNUDC and regulates its expression and activity. More research is required to examine the effect of miR-194 on cellular growth, shape and function, especially in clinical cancerous cells, in order to determine its therapeutic potential in regulating aberrantly expressed hNUDC, especially in downregulated hNUDC-related tumors.

\section{Acknowledgments}

The authors would like to acknowledge funding attributed to this study, namely by the National Natural Science Foundation of China (grant no. 31271230).

\section{References}

1. Koutsilieris M: Osteoblastic metastasis in advanced prostate cancer. Anticancer Res 13: 443-449, 1993.

2. Chambers AF, Groom AC and MacDonald IC: Dissemination and growth of cancer cells in metastatic sites. Nat Rev Cancer 2: 563-572, 2002.

3. Valastyan S and Weinberg RA: Tumor metastasis: Molecular insights and evolving paradigms. Cell 147: 275-292, 2011.

4. Hanahan D and Weinberg RA: Hallmarks of cancer: The next generation. Cell 144: 646-674, 2011.

5. Bagnyukova TV, Pogribny IP and Chekhun VF: MicroRNAs in normal and cancer cells: A new class of gene expression regulators. Exp Oncol 28: 263-269, 2006.

6. Iorio MV and Croce CM: MicroRNAs in cancer: Small molecules with a huge impact. J Clin Oncol 27: 5848-5856, 2009.

7. Lu J, Getz G, Miska EA, Alvarez-Saavedra E, Lamb J, Peck D, Sweet-Cordero A, Ebert BL, Mak RH, Ferrando AA, et al: MicroRNA expression profiles classify human cancers. Nature 435: 834-838, 2005.

8. Bartel DP: MicroRNAs: Target recognition and regulatory functions. Cell 136: 215-233, 2009.

9. Volinia S, Calin GA, Liu CG, Ambs S, Cimmino A, Petrocca F, Visone R, Iorio M, Roldo C, Ferracin M, et al: A microRNA expression signature of human solid tumors defines cancer gene targets. Proc Natl Acad Sci USA 103: 2257-2261, 2006.

10. Nicoloso MS, Spizzo R, Shimizu M, Rossi S and Calin GA: MicroRNAs-the micro steering wheel of tumour metastases. Nat Rev Cancer 9: 293-302, 2009.

11. Hurst DR, Edmonds MD and Welch DR: Metastamir: The field of metastasis-regulatory microRNA is spreading. Cancer Res 69: 7495-7498, 2009.

12. Jerónimo C, Bastian PJ, Bjartell A, Carbone GM, Catto JW, Clark SJ, Henrique R, Nelson WG and Shariat SF: Epigenetics in prostate cancer: Biologic and clinical relevance. Eur Urol 60: 753-766, 2011.

13. Porkka KP, Pfeiffer MJ, Waltering KK, Vessella RL, Tammela TL and Visakorpi T: MicroRNA expression profiling in prostate cancer. Cancer Res 67: 6130-6135, 2007.

14. Shen MM and Abate-Shen C: Molecular genetics of prostate cancer: New prospects for old challenges. Genes Dev 24: 1967-2000, 2010.

15. Coppola V, De Maria R and Bonci D: MicroRNAs and prostate cancer. Endocr Relat Cancer 17: F1-F17, 2010.

16. Chiang Y, Song Y, Wang Z, Liu Z, Gao P, Liang J, Zhu J, Xing C and $\mathrm{Xu} \mathrm{H}$ : microRNA-192, -194 and -215 are frequently downregulated in colorectal cancer. Exp Ther Med 3: 560-566, 2012.

17. Senanayake U, Das S, Vesely P, Alzoughbi W, Fröhlich LF, Chowdhury P, Leuschner I, Hoefler G and Guertl B: miR-192, miR-194, miR-215, miR-200c and miR-141 are downregulated and their common target ACVR2B is strongly expressed in renal childhood neoplasms. Carcinogenesis 33: 1014-1021, 2012.

18. Meng Z, Fu X, Chen X, Zeng S, Tian Y, Jove R, Xu R and Huang W: miR-194 is a marker of hepatic epithelial cells and suppresses metastasis of liver cancer cells in mice. Hepatology 52: 2148-2157, 2010.

19. Zhai H, Karaayvaz M, Dong P, Sakuragi N and Ju J: Prognostic significance of miR-194 in endometrial cancer. Biomark Res 1: $1,2013$.

20. Song Y, Zhao F, Wang Z, Liu Z, Chiang Y, Xu Y, Gao P and $\mathrm{Xu} \mathrm{H}$ : Inverse association between miR-194 expression and tumor invasion in gastric cancer. Ann Surg Oncol 19 (Suppl 3): S509-S517, 2012.

21. Wang B, Shen ZL, Gao ZD, Zhao G, Wang CY, Yang Y, Zhang JZ, Yan YC, Shen C, Jiang KW, et al: MiR-194, commonly repressed in colorectal cancer, suppresses tumor growth by regulating the MAP4K4/c-Jun/MDM2 signaling pathway. Cell Cycle 14: 1046-1058, 2015

22. Dong P, Kaneuchi M, Watari H, Hamada J, Sudo S, Ju J and Sakuragi N: MicroRNA-194 inhibits epithelial to mesenchymal transition of endometrial cancer cells by targeting oncogene BMI-1. Mol Cancer 10: 99, 2011. 
23. Gocke CD, Osmani SA and Miller BA: The human homologue of the Aspergillus nuclear migration gene $n u d C$ is preferentially expressed in dividing cells and ciliated epithelia. Histochem Cell Biol 114: 293-301, 2000.

24. Zhang MY, Huang NN, Clawson GA, Osmani SA, Pan W, Xin P, Razzaque MS and Miller BA: Involvement of the fungal nuclear migration gene $n u d C$ human homolog in cell proliferation and mitotic spindle formation. Exp Cell Res 273: 73-84, 2002.

25. Morris SM, Albrecht U, Reiner O, Eichele G and Yu-Lee LY: The lissencephaly gene product Lisl, a protein involved in neuronal migration, interacts with a nuclear movement protein, NudC. Curr Biol 8: 603-606, 1998 .

26. Aumais JP, Tunstead JR, McNeil RS, Schaar BT, McConnell SK, Lin SH, Clark GD and Yu-Lee LY: NudC associates with Lis1 and the dynein motor at the leading pole of neurons. J Neurosci 21: RC187, 2001.

27. Aumais JP, Williams SN, Luo W, Nishino M, Caldwell KA Caldwell GA, Lin SH and Yu-Lee LY: Role for NudC, a dynein-associated nuclear movement protein, in mitosis and cytokinesis. J Cell Sci 116: 1991-2003, 2003.

28. Zhou T, Aumais JP, Liu X, Yu-Lee LY and Erikson RL: A role for Plk1 phosphorylation of NudC in cytokinesis. Dev Cell 5: 127-138, 2003.

29. Miller BA, Zhang MY, Gocke CD, De Souza C, Osmani AH, Lynch C, Davies J, Bell L and Osmani SA: A homolog of the fungal nuclear migration gene $n u d C$ is involved in normal and malignant human hematopoiesis. Exp Hematol 27: 742-750, 1999.

30. Gocke CD, Reaman GH, Stine C, Zhang MY, Osmani SA and Miller BA: The nuclear migration gene NudC and human hematopoiesis. Leuk Lymphoma 39: 447-454, 2000.

31. Hartmann TB, Mattern E, Wiedemann N, van Doorn R, Willemze R, Niikura T, Hildenbrand R, Schadendorf D and Eichmüller SB: Identification of selectively expressed genes and antigens in CTCL. Exp Dermatol 17: 324-334, 2008.

32. Suzuki SO, McKenney RJ, Mawatari SY, Mizuguchi M, Mikami A, Iwaki T, Goldman JE, Canoll P and Vallee RB: Expression patterns of LIS1, dynein and their interaction partners dynactin, NudE, NudEL and NudC in human gliomas suggest roles in invasion and proliferation. Acta Neuropathol 113 591-599, 2007.

33. Hatakeyama H, Kondo T, Fujii K, Nakanishi Y, Kato H, Fukuda S and Hirohashi S: Protein clusters associated with carcinogenesis, histological differentiation and nodal metastasis in esophageal cancer. Proteomics 6: 6300-6316, 2006

34. Elbashir SM, Harborth J, Lendeckel W, Yalcin A, Weber K and Tuschl T: Duplexes of 21-nucleotide RNAs mediate RNA interference in cultured mammalian cells. Nature 411: 494-498, 2001

35. Lin SH, Nishino M, Luo W, Aumais JP, Galfione M, Kuang J and Yu-Lee LY: Inhibition of prostate tumor growth by overexpression of NudC, a microtubule motor-associated protein. Oncogene 23: 2499-2506, 2004

36. Livak KJ and Schmittgen TD: Analysis of relative gene expression data using real-time quantitative PCR and the $2^{-\Delta \Delta C T}$ Method. Methods 25: 402-408, 2001.

37. Lewis BP, Burge CB and Bartel DP: Conserved seed pairing, often flanked by adenosines, indicates that thousands of human genes are microRNA targets. Cell 120: 15-20, 2005.
38. Lewis BP, Shih IH, Jones-Rhoades MW, Bartel DP and Burge CB: Prediction of mammalian microRNA targets. Cell 115: 787-798, 2003.

39. Betel D, Koppal A, Agius P, Sander C and Leslie C: Comprehensive modeling of microRNA targets predicts functional non-conserved and non-canonical sites. Genome Biol 11: R90, 2010.

40. John B, Enright AJ, Aravin A, Tuschl T, Sander C and Marks DS Human MicroRNA targets. PLoS Biol 2: e363, 2004.

41. Enright AJ, John B, Gaul U, Tuschl T, Sander C and Marks DS: MicroRNA targets in Drosophila. Genome Biol 5: R1, 2003.

42. Faircloth LM, Churchill PF, Caldwell GA and Caldwell KA: The microtubule-associated protein, NUD-1, exhibits chaperone activity in vitro. Cell Stress Chaperones 14: 95-103, 2009.

43. Xiang X, Osmani AH, Osmani SA, Xin M and Morris NR: NudF, a nuclear migration gene in Aspergillus nidulans, is similar to the human $L I S-1$ gene required for neuronal migration. Mol Biol Cell 6: 297-310, 1995.

44. Pan RM, Yang Y, Wei MX, Yu XB, Ge YC and Xu P: A microtubule associated protein (hNUDC) binds to the extracellular domain of thrombopoietin receptor (Mpl). J Cell Biochem 96: 741-750, 2005.

45. Wei MX, Yang Y, Ge YC and Xu P: Functional characterization of hNUDC as a novel accumulator that specifically acts on in vitro megakaryocytopoiesis and in vivo platelet production. J Cell Biochem 98: 429-439, 2006.

46. Tang YS, Zhang YP and Xu P: hNUDC promotes the cell proliferation and differentiation in a leukemic cell line via activation of the thrombopoietin receptor (Mpl). Leukemia 22: 1018-1025, 2008.

47. Zhang C, Shu L, Kim H, Khor TO, Wu R, Li W and Kong AN: Phenethyl isothiocyanate (PEITC) suppresses prostate cancer cell invasion epigenetically through regulating microRNA-194. Mol Nutr Food Res 60: 1427-1436, 2016.

48. Selth LA, Townley SL, Bert AG, Stricker PD, Sutherland PD, Horvath LG, Goodall GJ, Butler LM and Tilley WD: Circulating microRNAs predict biochemical recurrence in prostate cancer patients. Br J Cancer 109: 641-650, 2013.

49. Sundaram P, Hultine S, Smith LM, Dews M, Fox JL, Biyashev D, Schelter JM, Huang Q, Cleary MA, Volpert OV, et al: p53-responsive miR-194 inhibits thrombospondin-1 and promotes angiogenesis in colon cancers. Cancer Res 71: 7490-7501, 2011.

50. Chen X, Wang Y, Zang W, Du Y, Li M and Zhao G: miR-194 targets RBX1 gene to modulate proliferation and migration of gastric cancer cells. Tumour Biol 36: 2393-2401, 2015.

51. Xu J, Kang Y, Liao WM and Yu L: MiR-194 regulates chondrogenic differentiation of human adipose-derived stem cells by targeting Sox5. PLoS One 7: e31861, 2012.

52. Han K, Zhao T, Chen X, Bian N, Yang T, Ma Q, Cai C, Fan Q, Zhou Y and Ma B: microRNA-194 suppresses osteosarcoma cell proliferation and metastasis in vitro and in vivo by targeting CDH2 and IGF1R. Int J Oncol 45: 1437-1449, 2014. 